\title{
NASA/CRUSTAL DYNAMICS PROJECT RESULTS: Tectonic Plate Motion Measurements with Mark-III VLBI
}

\author{
J. W. Ryan and T. A. Clark (for the CDP VLBI Group) \\ NASA/GSFC \\ Code 621.9 \\ Greenbelt, MD 20771
}

\begin{abstract}
The NASA Crustal Dynamics Project (CDP) has been using VLBI on intercontinental baselines to measure tectonic plate motions since 1979. We report on measurements between sites on the North American plate (Haystack/Westford, MA; Owens Valley and Mojave, CA; Ft. Davis, TX and Gilmore Creek, AK), the Eurasian plate (Onsala, Sweden; Wettzell, West Germany, and Shanghai, China), the Pacific plate (Kauai, HI; Kwajalein in the Marshall Islands, and Vandenberg AFB, CA), the African plate (Hartebesthoek, RSA), and Japan (Kashima).

The Mass.-Sweden baseline has been measured over 100 times as part of the CDP, MERIT, POLARIS, and IRIS projects. Since 1984, measurements to Europe have also included a new 18-m antenna at Wettzell, FRG. In 1984, the CDP began GAPE (the Great Alaska and Pacific Experiment) to measure motions of sites on the Pacific plate using our existing 9-m VLBI antenna at Vandenberg AFB plus new VLBI installations at the 9-m NASA antenna on Kauai and on a 26-m antenna at Kwajalein. At the same time, we also implemented a dedicated Alaskan VLBI station on a 26-m antenna at Gilmore Creek, near Fairbanks; the GAPE measurements have also included Mojave, Kashima, Westford, Onsala, and Wettzell. Selected CDP VLBI results are summarized in the following table along with a comparison to predictions of the Minster-Jordan RM-2 relative plate motion model.
\end{abstract}


Baseline

$$
\begin{aligned}
& \text { Observed RM-2 } \\
& \text { Rate Sigma Prediction } \\
& (\mathrm{mm} / \mathrm{yr}) \quad(\mathrm{mm} / \mathrm{yr}) \quad(\mathrm{mm} / \mathrm{yr})
\end{aligned}
$$

\begin{tabular}{|c|c|c|c|}
\hline $\begin{array}{l}\text { Westford-Onsala } \\
\text { Westford-Wettzell }\end{array}$ & $\begin{array}{l}16 \\
13\end{array}$ & $\begin{array}{l}3 \\
5\end{array}$ & $\begin{array}{l}17 \\
19\end{array}$ \\
\hline \multicolumn{4}{|c|}{ North American-Pacific Baselines } \\
\hline $\begin{array}{l}\text { Gilmore Creek-Vandenberg } \\
\text { Gilmore Creek-Kauai } \\
\text { Gilmore Creek-Kwajalein } \\
\text { Mojave-Vandenberg } \\
\text { Mojave-Kauai } \\
\text { Mojave-Kwajalein }\end{array}$ & $\begin{array}{r}-56 \\
-53 \\
-36 \\
20 \\
8 \\
-2\end{array}$ & $\begin{array}{r}6 \\
9 \\
7 \\
7 \\
7 \\
13\end{array}$ & $\begin{array}{r}-60 \\
-52 \\
-23 \\
22 \\
16 \\
23\end{array}$ \\
\hline \multicolumn{4}{|c|}{ Baselines to Japan } \\
\hline $\begin{array}{l}\text { Gilmore Creek-Kashima } \\
\text { Mojave-Kashima } \\
\text { Kauai-Kashima } \\
\text { Vandenberg-Kashima } \\
\text { Kwajalein-Kashima }\end{array}$ & $\begin{array}{l}-15 \\
-20 \\
-78 \\
-61 \\
-77\end{array}$ & $\begin{array}{r}8 \\
12 \\
11 \\
26 \\
9\end{array}$ & $\begin{array}{r}-7^{*} \\
-9^{*} \\
-87^{*} \\
-51^{*} \\
-94^{*}\end{array}$ \\
\hline
\end{tabular}

North American-European Baselines

Selected Intraplate Measurements

Gilmore Creek-Mojave

Kauai-Vandenberg

Kauai-Kwajalein

Vandenberg-Kwajalein

Westford-Owens Valley

Westford-Mojave

Mojave-Owens Valley

$\begin{array}{rrr}-12 & 4 & 0 \\ -15 & 9 & 0 \\ -7 & 8 & 0 \\ -20 & 18 & 0 \\ 2 & 2 & 0 \\ 0 & 4 & 0 \\ -3 & 2 & 0\end{array}$

* Assuming Kashima is on the Eurasian plate. 